Redaktoure fur:

\section{Redaktionsstab des Chemischen Zentralblattes}

Teil A : Dr. Eugen Klever, Dr. Armand Blaschette, Dr. Horst Llepack, Dipl.-lng. Ruth Mattner, Dipl.-Phys. Herbert Weber, Dr.-Ing. Christian Weiske. Wissenschaftliche Redaktionsmitglieder: Prof. Dr.-Ing. Bertold Reuter, Dr. Gerhard Schmidt

Teil B: Dr. Eugen Herr, Dr.-Ing. Gunther Pötzscher, Dipl.Ing. Joachim Richter, Dr.-Ing. Wolfgang Schramm

Teil C: Dr. Eugen Herr, Dipl-Chem. Peter Golinske, Dr. Irmtraut Hahn, Dipl.-Chem. Wolfgang Liebscher, Dr. Horst Pagel, Dr.-Ing. Ilse Spaeth. Wissenschaftliches Redaktionsmitglied: Dr. Margareta Boit. A 'Benredaktion: Prof. Dr. Otto Wichterle, Prag

Teil D: Dr. Eugen Herr, Dipl.-Chem. Heinz Czech. AuBenredaktion: Prof. Dr. Otto Wichterle, Prag

Teil E: Dr. Paul Loch, Dr. Willi Barz, Dr. Else Brandt, Dipl.-Chem. Peter Gregorzewski, Dr. Wolfgang Herrmann, Dr. Wilfried Hiller, Dipl.-Ing. Heinz Kockert, Dipl.-Biol. Margot Metze

Teil F: Dr. Willi Barz, Dipl.-Ing. Frigga Thomas

Teil G: Dr. Eugen Klever, Dr. Helene Frohllch

Teil H: Dr. Helga Volz, Dr. Gerd Bauer, Dipl.-Chem. Heinz Czech, Dr. Mechthilde Gehlen-Keller, Dipl.-Min. Hildtraut Noack, Dipl.-Ing. Gerhard Reisner, Dipl.-Ing. Frigga Thomas, Dr. Michael Winiker. Wissenschaftliches Redaktionsmital.: Dr.-Ing. Rudolf Karl Maller. Außenredaktion: Dr. Ingeborg Kalzendorf, Leuna
Register: Dr. Walther Schicke, Dr. Else Brandt. Dr. Helene Fröhlich, Dr. Margret Hänel, Gertrud Klesse, Dr. Gerda von Krueger, Dr.-Ing. Ruth Levi, Dr.-Ing. Günther Pötzscher, Dipl.-Chem. Wolfgang Răck, Dipl.-Chem. Asta Relsner, Dipl.-Ing. Elfrlede Rosdorff, Dr.-Ing. Wolfgang Schramm, Dr.-Ing. Nikolaus Ullmann, Dr. Irma Wilke, Dr. Maria Winter. Unter weiterer Mitarbeit von: Dr. Else Arnold, Dr. Christa Bahr, Dipl.-Chem. Ingrid Besser, Dipl.-Ing. Ingeborg Buchta, Dipl.-Chem. Sigrid Dehmlow, Dr. Irmgard Delius, Dipl.-Ing. Fritz Ehrhardt, Dipl.-Chem. Gertraud Herper, Dr. Wolfgang Herrmann, Dipl.-Ing. Werner Hoffmann, Dipl.-Chem. Eva Hunger, Dipl.-Chem. Ursula Krüger, Dipl.-Chem. Gunther Lietz, Dipl.-Ing. Gisela Maier, Dr.-Ing.Friedrich Meyer-Wildhagen,Dr.Steffl Mohrmann, Dipl.-Ing. Marietta Moldenschardt, Dipl.-Chem. Sylvia Oeisner, Dr. Horst Pagel, Dipl.Chem. Rosemarle Pankow, Dipl.-Ing. Brigitte Repp, Margret Rodegast, Dipl.-Chem. Klaus Schmidt, Dr. Ingeborg Stōrig. Dipl.-Chem. Brigitte Strauß, Dr. Edit Ulbrich, Dipl.-Chem. Irmela Wendtlandt, Dipl.-Chem. Rosemarie Werner

Entwicklungsabtellung:

Dr. Gerd Bauer, Dipl.-Ing. Fritz Ehrhardt

Linguistische Abteilung:

Slawische Dipl.-Chem. Else Heinemann, Dr. Helene Fröhlich, Dr. Georg Froelich, Dr. Eugen Klever, Joachim Sprachen:

Lehmberg, Prof. Dr. Max Ulmann, Dr.-Ing. Christian Welske

Unter weiterer Mitarbeit von: Ing. Sergej Baryschnikow, Dipl.-Ing. Otto Lebtag

AuBenredaktion: Dr. Hans Schulz, Leipzig

Fernöstliche Sprachen: Außenredaktion: Prof. Dr. Eturo Maekawa, Japan; Prof. Dr. Leonhard Schnler, Heidelberg

Außenredaktionen :

Wochenhefte: prom. Chem. Csc. Vladimír Dadák, Brünn; Prof. Dr. Otto Wíchterle, Prag; Prof. Dr. Jenó Plank, Budapest; Prof. Dr. B. Kurtev, Sofla; Prof. Dr. Eturo Maekawa, Japan; Prof. Dr. Leonhard Schuler, Heidelberg. Dr. Hans Schulz, Leipzig; Dr. Ingeborg Kalzendorf, Leuna

Patente: $\quad$ Dr. Julius Gante, Dr.-Ing. Hermann Brăucker, Dr. Hanns Donle, Dr. Walther Ganzlin, Dr.-Ing. Josef Muhleisen . Dr.-Ing. Hans-Jürgen Nitzschke, Dr.-Ing. Fritz Vier

Stándiger wissenschaftlleher Berater bei der Redaktionsleitung: Dr. Willi Barz

Referentendienst: Alice Hawelek, Berlin N 4, Schiffbauerdamm 19

Dr. Armand Blaschette, Berlin 30, Geisbergstraße 38

\title{
Sprachenbezeichnung in den Referaten des Chemischen Zentralblattes
}

(Abkürzungen laut DIN-Normblatt 1502, Beiblatt 1)

Albanisch = alb.
Bulgarisch $=$ bulg.
Chinesisch $=$ chin.
Dänisch $=$ dän.
Deutsch $=$ dt.
Englisch $=$ engl.
Estnisch = estn.
Finnisch $=$ finn.
Flämisch $=$ fläm.
Französisch = franz

Griechisch $=$ griech.

Irisch

Italienisch

$=\mathrm{ir}$.

Japanisch

$=$ ital.

Lateinisch

= jap.

Litauisch

= lat.

= litau.

Niederländisch $=$ niederl.

Norwegisch $=$ norw

Persisch = pers.

Polnisch

Portugiesisch $=$ port.
Rumänisch = rum.

Russisch = russ.

Schwedisch = schwed.

Serbokroatisch $=$ serbokroat.

Slowakisch = slowak.

Slowenisch = slowen.

Spanisch = span.

Tschechisch $=$ tscheoh

Türkisch = türk.

Ukrainisch = ukrain.

Ungarisch = ung. 


\title{
CHEMISCHES ZENTRALBLATT
}

VOLLSTÄNDIGES REPERTORIUM

FU'R ALLE ZWEIGE DER REINEN UND ANGEWANDTEN CHEMIE

1830 gegründet

1897-1945 von der Deutschen Chemischen Gesellschaft fortgeführt

Im Auftrage der Deutschen Akademie der Wissensehaften zu Berlin, der Chemischen Gesellschaft in der DDR, der Akademie der Wissenschaften zu Göttingen und der Gesellsehaft Deutscher Chemiker (in der BRD) herausgegeben von

Prof. Dr. Heinrich Bertsch und Prof. Dr. Dr.h. c. Wilhelm Klemm

\author{
134. JAHRGA NG
}

1963

SEITE $21405-22970$

Chefredakteure: Dr. Eugen Klever und Prof. Dr.-Ing. Eberhard Lelbnitz

1963

AKA DEMIE-YERLAG GMBH - BERLIN

VERLAG CHEMIE・GMBH・WEINHEIMIBERGSTR. 


\begin{tabular}{lcr} 
Heft-Nr. & Seitenzahl & Ausgabedatum \\
\hline 49 & $21405-21796$ & 4. Dezember 1963 \\
50 & $21797-22188$ & 11. Dezember 1963 \\
51 & $22189-22572$ & 18. Dezember 1963 \\
52 & $22573-22970$ & 25. Dezember 1963
\end{tabular}

Heranggeber: Prof. Dr. Heinrich Bertsch, Berlin, u. Prof. Dr. Dr. h. c. Wilhelm Klemm, Münster. Chefredakteure und verantwortlich tür den Inhalt: Dr. Eugen Klever, Berlin 30, Geisbergstr. 39, Ferneprecher: 249541, und Prof. Dr.-Ing. Eberhard Leibnitz, Berlin N 4, Schiffbauerdamm 19. Fernsprecher: 425571 .

(C)

Verlag: Akademie-Verlag GmbH. Berlin W 8, Leipziger Str. 3-4 (Fernsprecher: 2204 41, Telex-Nr.011 773, Postscheckkonto Berlin 35021), und Verlag Chemie, GmbH, Weinheim/Bergstr. (Fernsprecher: Weinheim 3685 [Sammelnummer]; Fernschreiber: 0465616 chemieverl wnh; Postscheckkonten: Frankfurt/M. $145314 \mathrm{u}$. Berlin [West] 7430). Bestellnummer: 1007/134. Das Chenische Zentralblatt erscheint wochentlich. Preis des Jahrganges einschl. Autoren-, Patent-, Sach- u. Formelregister: DM 1200,-; elnseltig bedruckte Ausgabe: DM 1350,-. Abgabe der Zeitschrift nur mit sămtlichen Registern. Abbestellungen nur bis spătestens 6 Wochen vor Ablauf des Kalenderjahres.

Alle Rechte, such die der Ubersetzung sowie der photomechanischen Wiedergabe, sind vorbehalten. All rights reserved (including those of translations into foreign languages). No part of this issue may be reproduced in any form, by photoprint, microfilm or any other means, without written permission from the publishers.

Die Wiedergabe von Gebrauchsnamen, Handelsnamen, Warenbezeichnungen usw, in diesen Heften berechtigt such ohne besondere Kennzeichnung nicht zu der Annahme, daB Bolche Namen im Sinne der Warenzelchen- und Markenschutz-Gesetzgebung als frei zu betrachten wären und daher von jedermann benutzt werden dürften.

Satz u. Druck: VEB Leipziger Druckhaus, Leipzig, III/18/203, 12/63.

Veroffentlicht unter der Lizenznummer 1273 des Presseamtes beim Vorsitzenden des Ministerrates.

$$
\text { Printed in Germany. }
$$

Die Anzeigenannahme erfolgt bei der DEWAG-WERBUNG, Berlin C 2, Rosenthaler Str. 28/31 und allen DEWAG-Betrieben in den Bexirksstädten der DDR sowie beim Verlag Chemio, GmbH, Weinheim/BergstraBe. 